synthesis at the level of $m \mathrm{RNA}$ transcription or translation? Although the answer to this question still remains obscure, some recent experiments have eliminated some of the possible mechanisms that have been proposed in the past.

Hall et al. (1964) found that most $T_{2} m \mathrm{RNA}$ species made early in infection (before the phage DNA replicates) are still present at late times when 'early' proteins are no longer being synthesized; this suggests regulation at the translational level. One, perhaps unlikely, possibility considered was that ribosomes by some modification are rendered incapable of binding 'early' $m$ RNA late in infection. But as Friesen, Dale and Bode (J. Mol. Biol., 28, 413; 1967) and Baldi and Haselkorn (J. Mol. Biol., 27, 193; 1967) have shown, this is not the case. Baldi and Haselkorn report briefly that about half the radioactivity given in a pulse at late times during $T_{4}$ infection is incorporated into 'early' $m \mathrm{RNA}$ and this binds to ribosomes equally as well as 'early' mRNA made at early times. Friesen et al. confirm this; they found 'early' $m$ RNA present at late times and associated with ribosomes just as it is early in infection. (The relative amounts of 'early' and 'late' $m$ RNA at late times remain debatable; Geiduschek et $a l$. (1966) and Kasai and Bautz (1967) claim the concentration of 'late' $m \mathrm{RNA}$ is 30 times that of 'early' mRNA.)

One explanation of these results is that although 'early' $m$ RNA at late times is associated with polysomes it is not translated. The 'early' messenger $m$ RNA itself may be altered, or part of the translating machinery other than the ribosomes may be modified; for example a new class of $t$ RNA made at late times may read codons restricted to early $m \mathrm{RNA}$ as missense or nonsense. Alternatively, since Greene and Korn (J. Mol. Biol., 28, 435; 1967) have now shown early that $m \mathrm{RNA}$ is unstable, and if the instability of $m \mathrm{RNA}$ results from its participation in translation, then 'early' $m$ RNA may be translated, albeit imperfectly, at late times and yield non-functional protein.

Greene and Korn measured the half-life of $T_{4} m \mathrm{RNA}$ at all stages during infection, using $\mathrm{C}^{14}$ uracil pulse labelling and actinomycin D to stop transcription, and found both 'early' and 'late' $m$ RNA has a half-life of $3.5 \mathrm{~min}$ at $37^{\circ} \mathrm{C}$. Furthermore, in the presence of actinomycin the rate of protein synthesis decays with the same half-life as the $m \mathrm{RNA}$. There was no evidence for stable $m$ RNA of any sort. Earlier this year Bose and Warren (Biochem. Biophys. Res.Commun., 26, 285; 1967) had reported that $m$ RNA for the early enzyme deoxythymidylate synthetase is unstable. These results eliminate Edlin's (1966) suggestion that the cessation of early protein synthesis occurs by competition, for the protein synthesizing machinery, between unstable 'early' $m$ RNA and accumulating stable 'late' $m$ RNA.

In the light of these experiments and given that at late times new species of $m$ RNA appear (Geiduschek et al., 1966), it seems likely that the regulatory process involves interplay between translational and transcriptional controls and there is ample evidence that in other systems the processes are interrelated.

\section{Induction of Meiosis}

from a Correspondent

NEW arrangements of genetic material arise from recombination and segregation of genes at meiosis.
Mitosis, however, serves to pass genetic material through many cell generations without any gene rearrangement. But what are the mechanisms which underlie the entry of a cell to the meiotic process from a previous mitotic way of life? An answer would help to explain how pairing of homologous chromosomes is brought about (for a cytogenetic approach see Riley and Chapman, Nature, 216, 60; 1967), and would ultimately enable genetic analysis of higher organisms to be carried out at will in cell culture.

Yeast cells can be induced to enter meiosis and sporogenesis by transferring them from a nutritionally rich growth medium to a poor, acetate-containing, sporulation medium. Some of the biochemical and cytological changes that occur during this switch from mitosis to meiosis have been published in two papers by Croes (Planta, 196, 209 and 227; 1967). The first visible indications of the onset of meiosis and spore development are an increase in nuclear and cell volume and cytoplasmic vacuolation, and are apparent two hours after transfer to the sporulation medium. Simultaneously there is a sharp increase in oxygen consumption and a small increase in protein content; but these, along with the RNA content, decline as spore formation continues. This lowering of metabolic activity is surprising since the gross morphological changes of sporogenesis, such as spore wall formation, occur 14 to 20 hours after transfer. Experiments with ethionine, which blocks both meiosis and spore maturation, showed that these processes are most readily blocked if the inhibitor is added during the first two hours after transfer, while less severe inhibition results if ethionine is given 4 to 6 hours after transfer. Further experiments showed that even before transfer to the sporulation medium the cells are prepared for meiosis. Ethionine given for two hours immediately before transfer caused a 99 per cent inhibition of subsequent meiosis and sporogenesis, while mitosis in that two hours was inhibited by only 26 per cent. Croes's experiments show that the potentiality for meiosis is latent in the cell at the time of transfer and it is contact with the acetate in the sporulation medium that causes the immediate induction of meiosis.

In the second paper Croes defines some of the metabolic patterns in the cell which correlate with the ability to enter meiosis. The number of meiotic cells induced is greatest if the transfer to sporulation medium is made when the culture is passing from the log to the stationary phase of growth. This transition occurs when glucose in the growth medium is exhausted by fermentation and the accumulated cthanol is then consumed in respiration through the glyoxylate cycle. This change from fermentation to respiration accounts for the stimulation of oxygen consumption by the sporulation medium as the acetate serves as the carbon source for the glyoxylate cycle. Although these metabolic changes appear significant in triggering meiosis, they seem far removed from the meiotic process itself. There are still many unanswered questions-does this radical change in cellular metabolism affect the types of RNAs and proteins synthesized? Are there special "meiotic" proteins ? How does the intracellular environment affect the behaviour of the nucleus? Finally, are there parallel changes in cells of higher organisms as they enter meiosis? A fusion of the biochemical and cytogenetic approach to meiosis should be very rewarding. 